\title{
Compound Tongluo Decoction promotes generation and homing of endothelial progenitor cells after cerebral infarction in rats by activating Shh signaling
}

\author{
Zhen Hui ${ }^{1}$, Wei $\mathbf{W u}^{2}$, Chengcheng $\mathrm{Xu}^{1}$, Sulei Wang${ }^{1}$, Jianxiang $\mathrm{Li}^{1}$, Jinghua \\ Zhang $^{1}$, Zhennian Zhang ${ }^{1 *}$ \\ ${ }^{1}$ Department of Neurology, Nanjing Hospital of Chinese Medicine Affiliated to Nanjing University of Chinese Medicine, ${ }^{2}$ Nanjing \\ University of Chinese Medicine, Nanjing, China \\ *For correspondence: Email: huizhen68@sohu.com
}

Sent for review: 12 February 2021

Revised accepted: 29 April 2021

\begin{abstract}
Purpose: To investigate the protective effects of Compound Tongluo decoction (CTD)on neurological function in rats, and the mechanism involved in its angiogenesis-promoting effect.

Methods: Rats were arbitrarily assigned to sham group, permanent middle cerebral artery infarction (PMCAO) group, and PMCAO+CTD group ( $P M C A O$ plus 7-day oral treatment with CTD). Neurological deficit scores and volume of stroke-damaged areas were measured after 7 days of treatment. The levels of bone marrow-derived endothelial progenitor cells (BMEPCs) in serum and brain tissues were determined by flow cytometry and immunofluorescence. The expression levels of sonic hedgehog (Shh), vascular endothelial growth factor (VEGF), and angiopoietin-1(Ang-1) at infarct sites and in BMEPCs were quantitated using western blot assay.

Results: The results showed that CTD markedly ameliorated neurological deficit, reduced volume of affected areas, and promoted the production and homing of BMEPCs. Moreover, CTD upregulated Shh, VEGF and Ang-1expressions at ischemic sites and EPCs, but promoted the proliferation and metastasis of EPCs. The CTD-induced changes were significantly suppressed by Shh inhibitor cyclopamine (CP). Conclusion: These results demonstrate that CTD promotes angiogenesis after cerebral infarction, probably by stimulating Shh signaling and triggering production and homing of EPCs, thereby providing neuroprotection against cerebral infarction. Thus, CTD is a potential neuroprotective agent against cerebral infarction in humans.
\end{abstract}

Keywords: Compound Tongluo decoction, Cerebral infarction, Angiogenesis, Endothelial progenitor cells, Sonic hedgehog

\begin{abstract}
This is an Open Access article that uses a fund-ing model which does not charge readers or their institutions for access and distributed under the terms of the Creative Commons Attribution License (http://creativecommons.org/licenses/by/4.0) and the Budapest Open Access Initiative (http://www.budapestopenaccessinitiative.org/read), which permit unrestricted use, distribution, and reproduction in any medium, provided the original work is properly credited.
\end{abstract}

Tropical Journal of Pharmaceutical Research is indexed by Science Citation Index (SciSearch), Scopus, International Pharmaceutical Abstract, Chemical Abstracts, Embase, Index Copernicus, EBSCO, African Index Medicus, JournalSeek, Journal Citation Reports/Science Edition, Directory of Open Access Journals (DOAJ), African Journal Online, Bioline International, Open-J-Gate and Pharmacy Abstracts

\section{INTRODUCTION}

Stroke is a neurological disease associated with high morbidity, mortality, and disability, and it imposes tremendous health and economic burden on the family and society. About $87 \%$ of stroke cases are diagnosed with ischemic stroke (also known as cerebral infarction). At present, the treatment of cerebral infarction is to restore blood reperfusion in the ischemic area as soon 
as possible. After restoring the supply of cerebral blood flow, the " ischemic penumbra " can be transformed into normal tissues, and the recovery of nerve function can be promoted [1]. The establishment of collateral circulation has been widely considered to be an effective way of improving blood perfusion. Vascular remodeling and angiogenesis are substantial bases for neurovascular unit (NVU) protection, neurofunctional remodeling, and establishment of collateral circulation in the ischemic area [2].

Endothelial progenitor cells (EPCs) derived from pluripotent stem cells are promising targets for angiogenesis, re-endothelialization, and blood vessel repair in various diseases. They are expected to be useful for revascularization due to their clinical values [3]. Evidence have shown that EPCs differentiate into mature endothelial cells (ECs) which play vital roles in angiogenesis after cerebral ischemic injury [4]. They also offer protection against endothelial injury and dysfunctional endothelium by directly incorporating themselves into newly formed blood vessels or stimulating pro-angiogenic growth factors or cytokines [5].

Compound Tongluo decoction (CTD) is a Traditional Chinese Medicine preparation. Several clinical validation studies have demonstrated its efficacy in the treatment of cerebral infarction. There is evidence that CTD boosts neurological function after acute cerebral infarction, and enhances ability to perform activities of daily life in patients [6]. A study in rats reported that it promoted micro-angiogenesis following cerebral infarction by increasing the expressions of VEGF and Ang-1, thereby providing neuroprotection against ischemic injury [7]. In the present study, the effect of CTD on EPC production and homing, as well as its relationship with the Shh signaling pathway were investigated in a pMCAO rat model and bone marrow-derived EPCs, as well as the mechanism of action.

\section{EXPERIMENTAL}

\section{Animals}

Male SPF Sprague-Dawley (SD) rats weighing 250 - $280 \mathrm{~g}$ were provided by Nanjing Drum Tower Hospital Affiliated to Medical College of Nanjing University. The rats were raised in the animal room of Nanjing Drum Tower Hospital under a $12 \mathrm{~h}$ dark-light cycle at controlled temperature of $20-23{ }^{\circ} \mathrm{C}$ and $40-60 \%$ relative humidity. Feed and water were supplied ad libitum. All experiments were approved by the Institutional Animal Welfare Ethics Committee of
Nanjing Drum Tower Hospital (approval no. 2019060312). The study followed the ethical principles of the National Institutes of Health Guide for the Care and Use of Laboratory Animals.

\section{Treatments}

The rats were arbitrarily assigned to sham group, pMCAO group, and pMCAO + CTD group. In the sham group, surgery was used to separate common, internal, and external carotid arteries (CCA, ICA and ECA) without ligation. The rats were intragastrically administered normal saline once daily for 7 days. In the pMCAO group, the rats underwent intragastric administration of normal saline once daily after pMCAO operation (ligation of the three mentioned arteries). Rats in the pMCAO + CTD group were orally administered CTD (Nanjing Hospital of Traditional Chinese Medicine) at a dose of 24 $\mathrm{g} / \mathrm{kg}$, once daily after establishment of pMCAO model. After 7 days of continuous treatment, all rats were euthanized, and brain tissue specimens were excised for further analysis.

\section{Establishment of pMCAO cerebral infarction model}

The rat model of pMCAO was established using a modified thread occlusion method described by Longa [8]. Following anesthesia with intraperitoneal sodium pentobarbital (1\%, 35 $\mathrm{mg} / \mathrm{kg}$ ), the rat CCA, ICA and ECA were surgically separated. The proximal ends of the CCA and ECA were ligated and closed, and the ICA was sutured using a thread. A small incision was made from the bifurcation to the upper end of the CCA ligation. Then, the MAC was blocked by inserting the tip round a single-thread nylon filament $(0.25-0.27 \mathrm{~mm})$ into the ICA through the CCA. The ICA ligation was available when the $18-22-\mathrm{mm}$ filament was inserted from the carotid bifurcation. After the tail end of the threaded bolt was cut, the incision was sutured layer by layer. The thread size was selected according to the weight and nutrition of each animal. All rats were kept at a mean temperature of $37^{\circ} \mathrm{C} \pm 0.5^{\circ} \mathrm{C}$ until post-operation monitoring.

\section{Isolation and transplantation of EPCs}

The isolation of EPCs, in vitro amplification and EPC culture were carried out as described earlier [9]. After extraction from SD rat tibia and femur, bone marrow monocytes were obtained through Ficoll density gradient centrifugation. The monocytes were cultured in EGM-2-MV medium on an ePetri chip coated with human fibronectin (Sigma, USA) to make them adhere to the 
sensor surface. They were cultured for 4 days, and non-adherent cells were washed off using PBS. The remnant monocytes were incubated in fresh medium for 7 days. The differentiated EPCs were fusiform-shaped and unavailable for isolation as attached cells. The viable second- or third-generation EPCs were prepared for further experiments after 14 days of culture.

The $50 \mu \mathrm{g} / \mathrm{ml}$ Dil cell-labeling solution (Molecular Probes, Inc., USA) was used to label ECPs. The CM-Dil-labeled EPCs was injected into rats through the tail vein. At the end of the animal experiments, EPC expressions at infarct sites were confirmed using inverted fluorescence microscopy.

\section{Evaluation of neurological function}

The Longa scale was used to evaluate neurological deficits in pMCAO rats at multiple time points. A score of 0 was suggestive of normal neurological function or no neurological impairment, while a score of 1 indicated inability to vertically extend the left front paw. Scores of 2 , 3 and 4 were for leaning and cycling to the left, falling to the left, and failure to walk spontaneously/disturbance of consciousness, respectively.

\section{Triphenyltetrazolium chloride (TTC) staining}

On day 7 after establishment of the pMCAO model, the rats were sacrificed under $1 \%$ pentobarbital anesthesia. Brain specimens were frozen and transversally sectioned into 2-mm slices which were placed in $2 \%$ TTC to protect from light, and incubated at $37^{\circ} \mathrm{C}$ for $30 \mathrm{~min}$. Gross observation of brain slices showed that normal brain tissue appeared red, while infarcted areas were pale. The brain tissue sections were fixed for $24 \mathrm{~h}$, and images were captured using a Sony Alpha 7R IV digital camera. The infarct volume induced by pMCAO was calculated as described elsewhere [10].

\section{Fluorescence activated cell sorting (FACS)}

Bone marrow samples (100 $\mu \mathrm{L})$ were incubated with PE-conjugated anti-rat CD34 monoclonal antibody (Santa Cruz, USA) and biotinconjugated anti-rat VEGFR2 monoclonal antibody (Novus Biologicals, USA) for $25 \mathrm{~min}$ in the dark. Red blood cell lysis buffer (Beyotime Technology Ltd., China) was added to the cell samples. After 5-min incubation, the cells were centrifuged at $1500 \mathrm{rpm}$ for $5 \mathrm{~min}$ at $4^{\circ} \mathrm{C}$, and rinsed in PBS. The EPCs were stained with Streptavidin FITC (eBioscience, USA) after 30min incubation, and the proportions of EPCs with double-positive staining for CD34 and VEGFR2 were calculated.

\section{Immunoblotting}

Brain samples of the ischemic cortex or cells were subjected to protein extraction by homogenizing in an ultrasound homogenizer. The supernatant was centrifuged in the cold for $10 \mathrm{~min}$ at $12000 \mathrm{~g}$, and protein content was measured using a BCA analysis kit. Equal amounts of protein were resolved on SDSpolyacrylamide gel electrophoresis and transferred to polyvinylidene difluoride membranes which were sealed by incubation with $5 \%$ fat-free milk solution, followed by incubation overnight at $4^{\circ} \mathrm{C}$ with primary antibodies i.e., rabbit anti-Shh $(1: 200)$, rabbit anti-Ang-1(1:200), rabbit anti-VEGF (1:200), and rabbit anti-GAPDH antibodies (1:500, Santa Cruz, USA). Thereafter, the membranes were incubated with HRP-conjugated anti-rabbit antibody (1:2000, ICN Pharmaceuticals) at room temperature for $2 \mathrm{~h}$. Protein bands were visualized and protein expressions levels of Ang1, Shh, VEGF were assessed using Image J software, with the expression of GAPDH as a loading control.

\section{MTT assay}

The effect of CTD on the proliferation of EPCs was determined using MTT assay kit (Sigma Aldrich). Following seeding of cells in 96-well plate $\left(5 \times 10^{3}\right.$ cells/well $), 10 \mu \mathrm{L}$ of MTT solution was added from day 1 to day 3 for continuous culture for $4 \mathrm{~h}$. Thereafter, the supernatant was discarded, and $200 \mu \mathrm{L}$ DMSO was added to each well to solubilize the formazan crystals formed. After incubation for 10 min under vortexstirring, the absorbance of each well was measured at $490 \mathrm{~nm}$ using a microplate reader (Thermo MK3, USA).

\section{Transwell migration assay}

The density of EPCs was adjusted to $1 \times 10^{5}$ cells $/ \mathrm{mL}$ after CTD or CTD+CP treatment, for 24 h. Transwell assay was used to measure cell migration in 24-Transwell plates (Costar, Corning Inc., USA). A suspension of EPCs $\left(1 \times 10^{5}, 200\right.$ $\mu \mathrm{L} /$ well) was added to the upper compartment of the Transwell chamber, while a culture medium containing $20 \%$ FBS was placed in the lower chamber. The EPCs were allowed to migrate for $15 \mathrm{~h}$, after which the migrated cells were fixed with methanol, and stained with crystal violet dye solution. The stained cells were photographed and counted in triplicate under light microscopy. 


\section{Statistics}

Values are expressed as mean \pm SD. Paired comparison was done using $t$-test, followed by Tukey's post hoc test. The level of statistical significance was set at $p<0.05$.

\section{RESULTS}

\section{CTD reduced cerebral infarction volume in} rats

The protective effect of CTD against ischemic injury following cerebral infarction was determined in terms of stroke volume in each group using TTC staining. The results showed that stroke volume was markedly reduced in pMCAO+CTD group, relative to pMCAO group ( $p$ $<0.05$; Figure $1 \mathrm{~A}$ ).
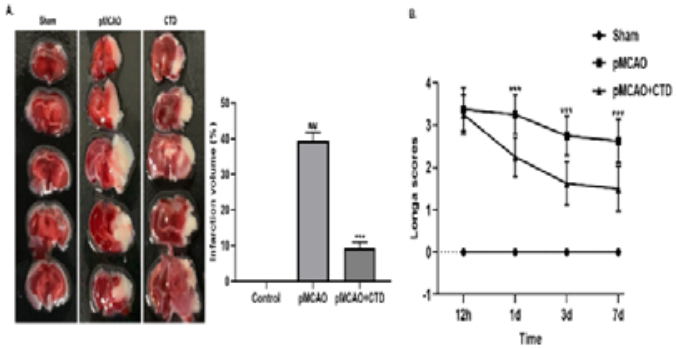

Figure 1: Effect of CTD on ischemic injury and neurological function following cerebral infarction. A. Lower neurological function score after CTD treatment. B. Volume of pMCAO-damaged areas, as determined using TTC staining. CTD treatment decreased the volume of damaged areas in pMCAO model. ${ }^{\#} P<0.05$, vs. sham; ${ }^{*} p<0.05$, vs. pMCAO; $\mathrm{n}=$ 8 per group

\section{CTD improved neurobehavioral ability of rats} with cerebral infarction

The neurological function of rats with cerebral infarction was determined at multiple time points to ascertain the effect of CTD on neurobehavioral ability. Longa's profile showed that rats in the PMCAO + CTD group achieved lower scores at $12 \mathrm{~h}$, day 1 , day 3 , and day 7 , when compared to rats in pMCAO group ( $p<0.05$; Figure $1 \mathrm{~B})$.

\section{CTD promoted mobilization and homing of BMEPCs in rats with cerebral infarction}

The level of BMEPCs in each group was determined flow cytometrically as an index of the effect of CTD on angiogenesis. The pMCAO group had a higher level of BMEPCs than the sham group $(p<0.05)$. However, BMEPCs level was higher in $P M C A O+C T D$ group than in pMCAO group ( $p<0.05$; Figure $2 A$ ). The level of EPCs at infarct sites was measured using immunofluorescence after injection of CM-Dillabeled BMEPCs. The pMCAO + CTD group exhibited the highest level of EPCs at infarct sites, followed by the pMCAO group and the sham group (Figure $2 \mathrm{~B}$ ).

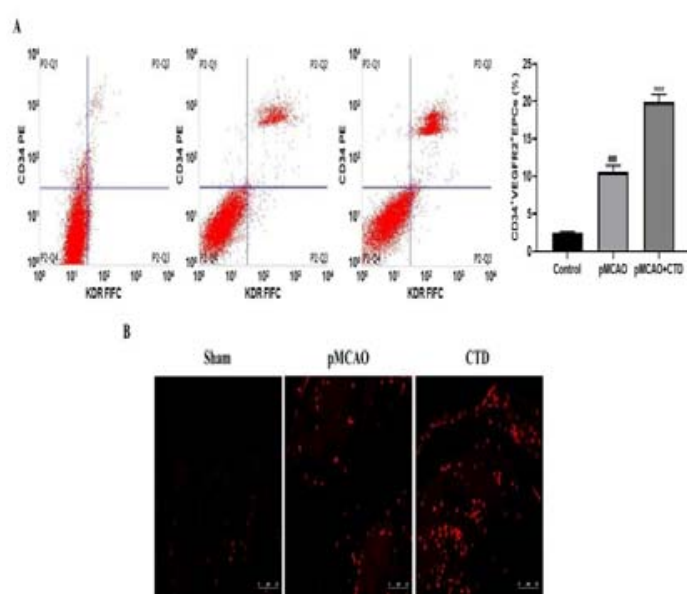

Figure 2: Effect of CTD mobilization and homing of BMEPCs in rats with cerebral infarction. A: Number of BMEPCs, as determined using flow cytometry. The number of CM-Dil-labeled cells was counted. B: number of BMEPCs at infarct sites, as determined using immunofluorescence. ${ }^{\#} p<0.05$, vs. sham; " $p<$ 0.05 , vs. pMCAO; $\mathrm{n}=3$ per group; Scale bar: $50 \mu \mathrm{m}$

\section{CTD stimulated Shh signaling and upregulated expressions of VEGF and Ang-I}

To reveal the mechanism through which CTD promoted angiogenesis in cerebral infarction, we used the WB analysis to detect the expression of Shh, VEGF and Ang-I. The pMCAO group had significantly up-regulated cerebral expressions of Shh, VEGF, and Ang-I, when compared with the sham group, while there were even higher expressions of Shh, VEGF and Ang-I in the brain in PMCAO+CTD group than in the PMCAO group $(p<0.05)$. These results are shown in Figure $3 \mathrm{~A}$ - C.
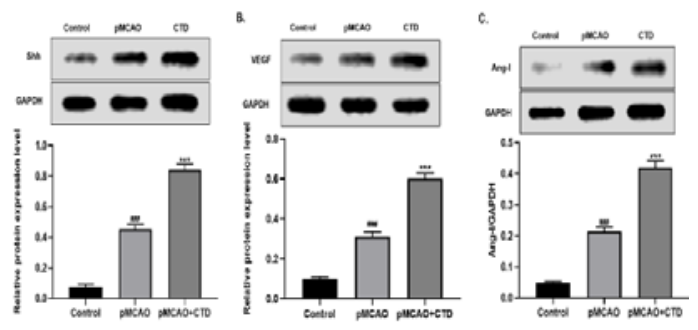

Figure 3: CTD activated the SHH signaling pathway and promoted the expressions of VEGF and Ang-I. AC. Quantification of cerebral Shh, VEGF, and Ang-I expressions using WB analysis. The experiment was 
repeated three times independently. ${ }^{\#} P<0.05$, vs. sham; ${ }^{*} p<0.05$, vs. pMCAO

\section{CTD enhanced amounts of VEGF and Ang-I in BMEPCs via activation of Shh signaling}

The Shh inhibitor CP was used to confirm the effect of Shh pathway activation in vitro. The results revealed that CTD markedly enhanced the expressions of Shh, VEGF, and Ang-I in EPCs of rats, relative to control $(p<0.05)$. However, this effect was potently repressed by $\mathrm{CP}(p<0.05)$. These results are shown in Figure $4 A-C$.

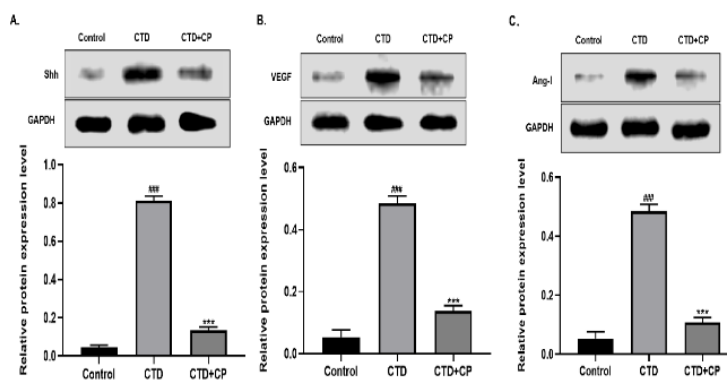

Figure 4: Effect of CTD expression levels of Ang-I and VEGF in BMEPCs. Quantification of expression levels of Shh, VEGF, and Ang-I in BMEPCs using WB (A-C). ${ }^{\#} p<0.05$, vs. control; ${ }^{*} p<0.05$, vs. CTD; $n=3$

\section{CTD boosted proliferation and migration of BMEPCs through Shh pathway activation}

The influence of CTD on proliferative and migratory potential of BMEPCs were determined in vitro. It was found that CTD triggered a high level of EPC activity, when compared to control $(p<0.05)$. In contrast, co-treatment with CTD and CP significantly suppressed the viability of EPCs $(p<0.05$; Figure 5 A). The Transwell assay showed that CTD markedly facilitated the migration of EPCs, but this was markedly suppressed with co-administration of CP $(p<$ 0.05). These results are shown on Figure $5 \mathrm{~B}$.
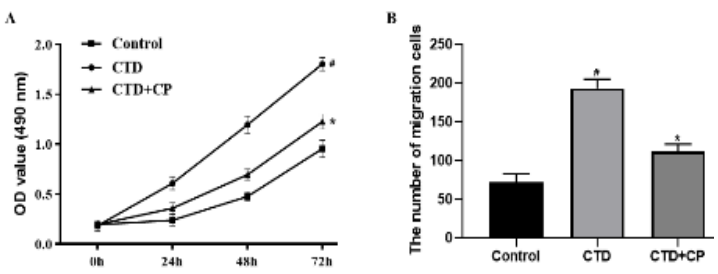

Figure 5: Effect of CTD on proliferation and migration of BMEPCs. A: cell viability of EPCs, as determined using MTT assay. B: migration of EPCs, as determined using Transwell migration assay. ${ }^{\#} P<$ 0.05 , vs. control; ${ }^{*} p<0.05$, vs. CTD; $\mathrm{n}=6$

\section{DISCUSSION}

Ischemic stroke caused by cerebral artery occlusion results in severely impaired blood supply to a specific region of the brain and cell death, rapid shrinkage of infarct areas, and neurological dysfunction. Local reperfusion at infarct sites is currently a popular strategy for treating these lesions so as to induce remission [10]. Interventions aimed at activating collateral circulation and anastomosis early may propel cerebral blood flow to ischemic areas, with continuous activation stimulating angiogenesis [11]. There is growing evidence indicating that angiogenesis produces extra blood flow for speedy microcirculation in infarct regions, thereby enhancing recovery of neurological function and reducing the volume of strokedamaged areas [12]. The results of this study showed that CTD significantly reduced pMCAOdamaged areas and improved neuromotor recovery, with higher levels of EPCs in peripheral blood and ischemic brain tissue at 1, 3, and 7 days after pMCAO. These results indicate that CTD provides neuroprotection against ischemic damage.

During early angiogenesis, VEGF, VEGFR-2, Ang-I, and Tie-2 are synchronously expressed on vessels from their formation to maturation [13]. Studies have shown that Shh signaling regulates amounts of Ang-1 and VEGF in ischemic areas, making it a common morphogenetic factor in neurodevelopment and angiogenesis during embryogenesis [14]. Several evidence have proved that Shh also mediates angiogenesis in postpartum life under pathological conditions $[15,16]$. Specifically, it produces strong angiogenetic effects via regulation of the Ang-1 and VEGF in interstitial mesenchymal cells [17]. In addition, it reduces the volume affected by MCAO by promoting angiogenesis after ischemia [18]. The present study has revealed that CTD markedly up-regulated the expressions of Shh, VEGF and Ang-I at pMCAO-affected areas in rats. It also stimulated the expressions of these proteins in BMEPCs in vitro. This study also revealed that CTD-induced promotion of the migratory and proliferative potential of BMEPCs in vitro were suppressed by the Shh inhibitor CP. Thus, CTD enhanced formation and migration of BMEPCs probably through activation of the Shh pathway and control of levels of VEGF and Ang1.

\section{CONCLUSION}

This study has shown that CTD enhances recovery of neurological function in pMCAO rats by reducing the volume of pMCAO-damaged 
regions. It facilitates angiogenesis by promoting the formation and homing of BMEPCs via activation of Shh pathway through control of the levels of VEGF and Ang-1. Overall, CTD offers effective neuroprotection against ischemic brain injury. Thus, the findings shed some light on the efficacy of CTD in cerebral infarction, and provide a basis for further investigations for enhanced neurological recovery after permanent middle cerebral artery infarction.

\section{DECLARATIONS}

\section{Acknowledgement}

The study was supported by the National Natural Science Foundation of China (no. 81703975), the Nanjing Medical Science and Technique Development Foundation (no. QRX17185), and the Priority Academic Program Development of Jiangsu Higher Education Institutions (no. 2019YSHL094).

\section{Conflict of interest}

No conflict of interest is associated with this work.

\section{Contribution of authors}

We declare that this work was performed by the authors named in this article and all liabilities pertaining to claims relating to the content of this article will be borne by the authors. Zhen Hui and Zhennian Zhang designed the study, Wei Wu and Chengcheng $\mathrm{Xu}$ supervised the data collection, and analyzed the data. Sulei Wang interpreted the data and prepared the manuscript for publication. Jianxiang Li and Jinghua Zhang supervised the data collection, analyzed the data and reviewed the draft of the manuscript.

\section{Open Access}

This is an Open Access article that uses a funding model which does not charge readers or their institutions for access and distributed under the terms of the Creative Commons Attribution License (http://creativecommons.org/licenses/by/ 4.0) and the Budapest Open Access Initiative (http://www.budapestopenaccessinitiative.org/rea d), which permit unrestricted use, distribution, and reproduction in any medium, provided the original work is properly credited.

\section{REFERENCES}

1. Paul S, Candelario-Jalil E. Emerging neuroprotective strategies for the treatment of ischemic stroke: An overview of clinical and preclinical studies. Exp Neurol 2021; 335: 113518.

2. Ginsberg $M D$. The cerebral collateral circulation: Relevance to pathophysiology and treatment of stroke. Neuropharmacol 2018; 134(Pt B): 280-292.

3. Wang ZC, Qi J, Liu LM, Li J, Xu HY, Liang B, Li B. Valsartan reduces AT1-AA-induced apoptosis through suppression oxidative stress mediated ER stress in endothelial progenitor cells. Eur Rev Med Pharmacol Sci 2017; 21: 1159-1168.

4. Chong MS, Ng WK, Chan JK. Concise Review: Endothelial Progenitor Cells in Regenerative Medicine: Applications and Challenges. Stem Cells Transl Med 2016; 5: 530-538.

5. Asahara $T$, Kawamoto A, Masuda $H$. Concise review: Circulating endothelial progenitor cells for vascular medicine. Stem Cells 2011; 29: 1650-1655.

6. Hui Z, Zhang JN, Zhang JH, Liu KJ, Xiao J, Li JB, Zheng $\mathrm{PH}$, Wang JQ. effects of Fufang Tongluo decoction on neurological function and vascular growth-related factors in patients with acute cerebral infarction. J Tradit Chin Med (Chin) 2018; 59:1383-1385.

7. Hui Z, Zhang JH, Shi ML, Wang JQ. The neuroprotection of compound Tongluo decoction on cerebral infarction in rats and its angiogenesis mechanism. Chin J Integr Med Cardio/Cerebrovascular Dis (Chin) 2018; 16:153-158.

8. Han L, Li J, Chen Y, Zhang M, Qian L, Chen Y, Wu Z, Xu $Y$, Li J. Human Urinary Kallidinogenase Promotes Angiogenesis and Cerebral Perfusion in Experimental Stroke. PLoS One 2015; 10: e0134543.

9. Luo T, Shu J, Lu Z, Han T, Fang G, Xue X. Potential Role of Caveolin-1 in Regulating the Function of Endothelial Progenitor Cells from Experimental MODS Model. Mediators Inflamm 2019; 2019: 1-13.

10. Li Y, LU Z, Keogh CL, YU SP, Wei L. Erythropoietininduced neurovascular protection, angiogenesis, and cerebral blood flow restoration after focal ischemia in mice. J Cereb Blood Flow Metab 2007; 27: 1043-1054.

11. Beck H, Plate KH. Angiogenesis after cerebral ischemia. Acta Neuropathol 2009; 117: 481-496.

12. Zhang RL, Chopp M, Roberts C, Liu X, Wei M, NejadDavarani SP, Wang $X$, Zhang ZG. Stroke increases neural stem cells and angiogenesis in the neurogenic niche of the adult mouse. PLoS One 2014; 9: e113972.

13. Palladino M, Gatto I, Neri V, Straino S, Silver M, Tritarelli A, Piccioni A, Smith RC, Gaetani E, Losordo DW, et al. Pleiotropic beneficial effects of sonic hedgehog gene therapy in an experimental model of peripheral limb ischemia. Mol Ther 2011; 19: 658-666.

14. Nagase $T$, Nagase $M$, Machida M, Fujita T. Hedgehog signalling in vascular development. Angiogenesis 2008; 11: 71-77.

15. Byrd N, Becker S, Maye $P$, Narasimhaiah R, St-Jacques $B$, Zhang $X$, McMahon J, McMahon A, Grabel L. Hedgehog is required for murine yolk sac angiogenesis. Development 2002; 129: 361-372. 
16. Lee SW, Moskowitz MA, Sims JR. Sonic hedgehog inversely regulates the expression of angiopoietin-1 and angiopoietin-2 in fibroblasts. Int $\mathrm{J} \mathrm{Mol} \mathrm{Med} \mathrm{2007;} \mathrm{19:}$ 445-451.

17. Pola R, Ling LE, Silver M, Corbley MJ, Kearney M, Blake Pepinsky R, Shapiro R, Taylor FR, Baker DP, Asahara, et al. The morphogen Sonic hedgehog is an indirect angiogenic agent upregulating two families of angiogenic growth factors. Nat Med 2001; 7: 706-711.

18. Huang SS, Cheng $H$, Tang CM, Nien MW, Huang YS, Lee IH, Yin JH, Kuo TB, Yang CC, Tsai SK, et al. Antioxidative, anti-apoptotic, and pro-angiogenic effects mediate functional improvement by sonic hedgehog against focal cerebral ischemia in rats. Exp Neurol 2013; 247: $680-688$ 